
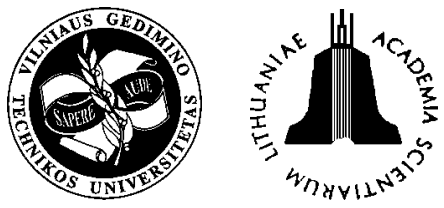

ISSN 1648-4142 TRANSPORT

http:/www.vtu.lt/english/editions

TRANSPORT - 2004, Vol XIX, No 2, 69-74

\title{
ROTARY MOTION TRANSMISSION AND STABILIZATION DEVICES
}

\author{
Marijonas Bogdevičius, Jurgis Jurevičius, Bronislovas Spruogis \\ Dept of Transport Technological Equipment, Faculty of Transport Engineering, Vilnius Gediminas Technical \\ University, Plytinès g. 27, LT-10105 Vilnius-16, Lithuania.E-mail: tti@ti.vtu.lt, \\ Tel: (+370 5) 2744783, fax: (+370 5) 2745060 \\ Received 2003-11-30; accepted 2004-01-15
}

\begin{abstract}
To connect rotors, to transmit and stabilize rotation motion a new class of rotary motion transmission and stabilization devices (RMTSD) was developed. They include the rings which deform axialy, radially and at an angle the radial segments, etc. with joined concentrated inertial, dissipative and elastic elements. RMTSD design schemes are copyrighted and covered by the patents of the USA, England and Germany.

The classification of RMTSD development is performed according to the following characteristic features: dynamic characteristics, kind of elastically creeping elements, peculiarities of design elements, fastening, materials, etc. The classification presented gives the possibility to develop lots of constructions of new RMTSD of this type.
\end{abstract}

Keywords: transmission, stabilization devices, coupling.

\section{Introduction}

The rotor system consists of many synchronously rotating links. Due to coupling links shafts misalignment, non-balanced parts, outside and exploitation disturbances and changes of the supplied energy, the links rotate irregularly. The above factors cause an increased dynamic load in machines and mechanisms, which gives rise to rotary vibration. When summed, these factors reach rather high values. For example, in mighty pumping stations of oil fields gear shaft misalignment reaches $2-4 \mathrm{~mm}$ of radial direction and up to $10 \mathrm{~mm}$ of axial direction.

The research was carried out how to decrease rotary vibrations and the forces which provoke them. One of the most effective ways of decreasing vibrations is the improvement of machine assembly construction elements and their change by constructions resistant to vibrations. Vibration dampers can be used for this effective RMTSD in the form of various clutches. Vibration protection in coupling devices manifests itself as suppression of vibration of constituent links of the elements [1-8].

Lately, two ways have been used to avoid undesirable harmful vibrations, i.e. the development of mechanisms with low activity vibrations and installing of special constructional assemblies which suppress and absorb vibrations in the machines.

In the rotor systems the elastic clutches with nonlinear characteristics are used most often. The clutches used up to now not fully satisfy the vibration protection requirements. They poorly suppress vibration and im- pacts. At present new clutch constructions, the action of which is based on the interaction between the forces of rotating masses and those of elastic elements, are worth of special attention. Due to this interaction, the radial stiffness of shaft coupling device (clutch) decreases. Thus, the development of new rotary motion transmission and stabilization devices, their metrical synthesis and optimization of elastic element parameters through optimal location and orientation of their elements in the constructions enables to obtain good characteristics of the devices as well as good indices of their exploitation.

RMTSD used as the clutches of rotary moment transfer do not meet the increasing requirements of industry. With decreasing dimensions of machines, increasing speed and productivity, the dynamic loads of some parts of machines increased greatly. For obtaining more precise machines, one must diminish the arisen harmful vibrations. Increasing speeds of machines impose heavy demands to RMTSD. They are: to compensate radial and angle misalignment of coupling shaft axes, to suppress impacts and interference, to isolate vibrations better, to avoid resonance swings or to move them away from a zone of machine working swings, to suppress dynamically rotary vibrations, to stabilize the rotary motion on the principle of sequence, etc. Besides, RMTSD must possess good exploitation properties at high temperatures and in the presence of dangerous chemical factors. They must be easily assembled, dismantled and repaired, steady, firm and reliable. 


\section{Classification of new developed RMTSD}

The author has developed more than two hundred design schemes of new devices which were copyrighted in the USSR and covered by the patents of the USA, England and Germany. More than 170 design schemes belong to RMTSD, clutches and rotary vibration dampers. According to their destination (Fig 1), RMTSD are classified as [1]:

- clutches for transmission and stabilization of rotary motion;

- vibration dampers for stabilization of rotary motion. According to the type of elastically creeping elements (ECE), the clutches are classified as clutches with homogeneous elastic elements (HEE), clutches with composite elastic elements (CEE), clutches with cord elements and clutches with continuum ECE.

Vibration dampers depending on the type of ECE can be shaped like a ring or like a band.
Depending on the shape of the elements being deformed clutches with ECE can be divided into 3 groups: clutches with ring elements, with band-string elements and with mixed elements. Clutches with ECE include lever, planetary and chain clutches. Clutches with cord elements can be with flat trapezium belts or type elastic elements.

The working space of clutches with continuum ECE can be filled with fluid, air or powder. Ring clutches can possess cutting rings, compact rings, composite rings, assembled rings and rings wound from a wire. More than 170 design schemes belong to band-string ECE can be with band elements, string elements and mixed bandstring elements. In the clutches with HEE can serve rings with ring sector elements, rings on elastic supports, chains pulled by cylinder springs, springy coupling chains, closed elastic contour of free shape, and various combinations of elastic elements.

Half-clutches of planetary clutches can be connected by friction or gear contact.

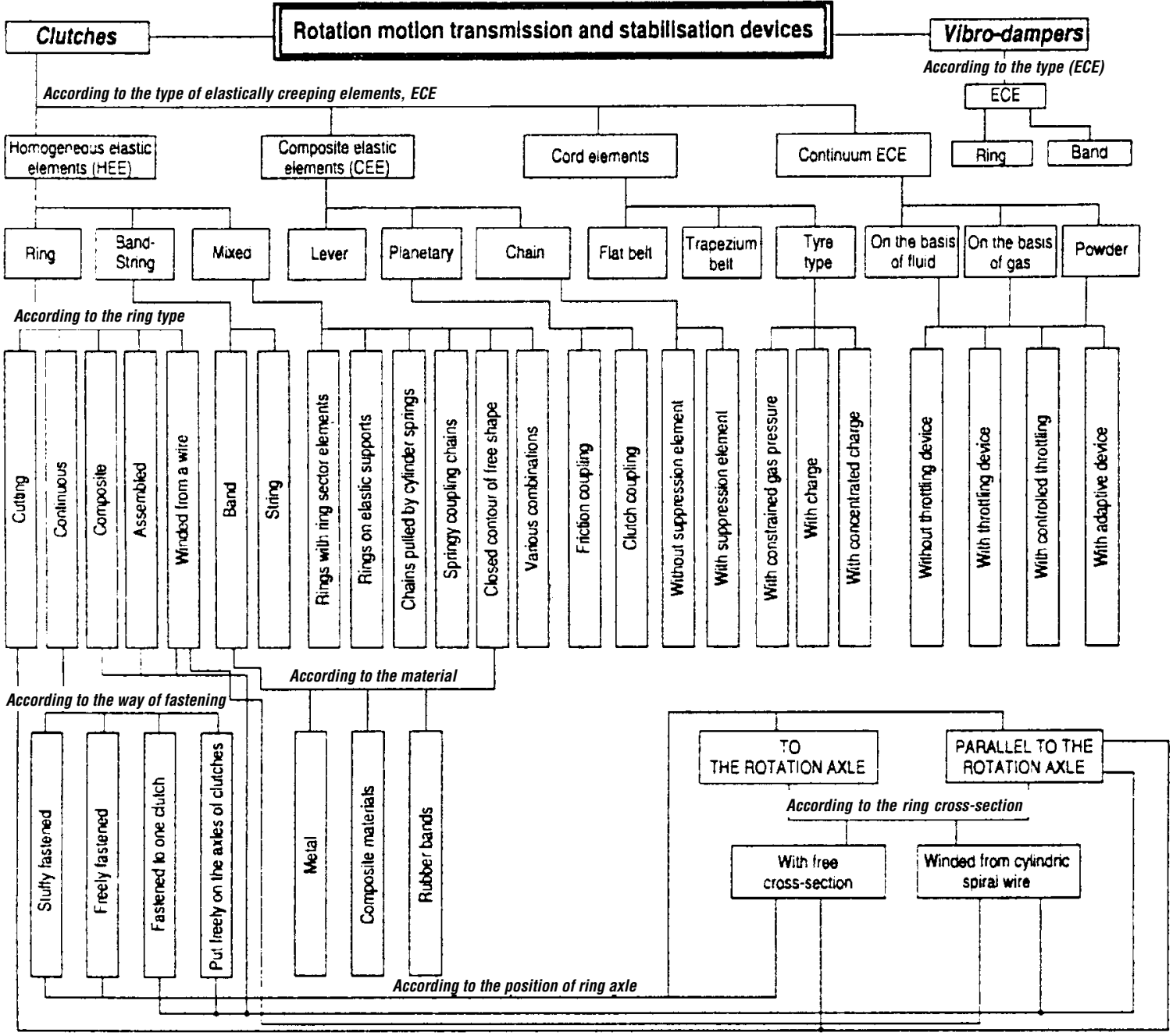

Fig 1. Classification of rotation motion transmission 
Chain clutches can be without a suppressing element which suppresses interferences and impacts and with it. Interferences and impacts show themselves through the inclination of a chain clutch coupling element (chain) at the moments of gear starting, reversing or stopping.

Clutches with continuum ECE can be without and with throttling devices, with controll throttling and adaptive control devices.

More detailed classification of clutches is shown in Fig 1.

More detailed information on RMTSD construction, characteristics and features is presented in [1].

\section{Elastic centrifugal ring clutches}

Elastic centrifugal ring clutches (ECRC) form a peculiar group of elastic centrifugal ring clutches.

The clutch (Fig 2) consists of driving half-clutch 1, driven half-clutch 2 , elastic ring 3 and loads 4 , which, when the clutch rotates, perform the role of centrifugal masses [2, 3].

Various ECRC design schemes are possible (Fig 3): with one (Fig 3 a) or several (Fig 3 b) elastic rings, with concentrated or distributed masses, with the masses located on separate rings (Fig $3 \mathrm{c}$ ); additional elastic chains connecting the rings with one two half-clutches (Fig $3 \mathrm{~d}$ ).

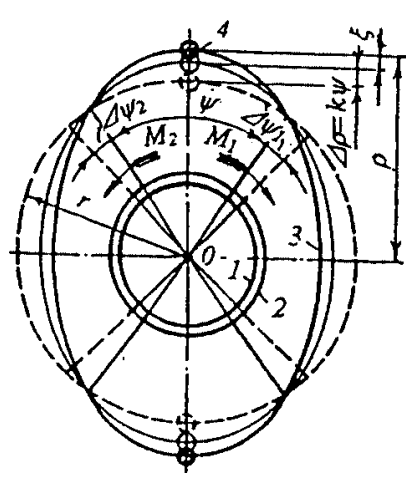

Fig 2. Principal scheme of the clutch
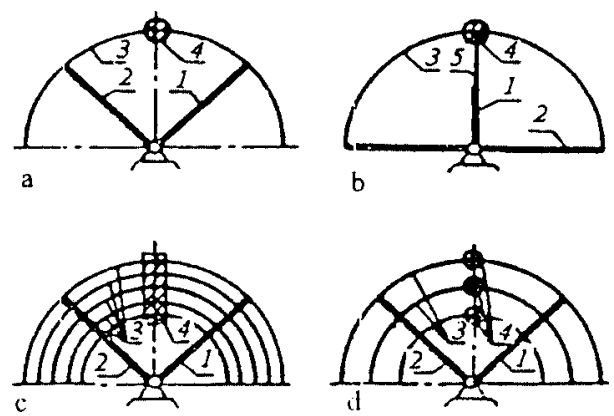

Fig 3. Example of ECRC design schemes
Explanations to Fig 3: 1 - driving half-clutch; 2 driven half-clutch; 3 - elastic rings; 4 - centrifugal masses; 5 - other elastic links.

The principle of the clutch functioning is based on the interaction of elastic restoring forces of the rotating ring being deformed with centrifugal forces of rotating masses.

All the system consists of axle mechanisms. To set its positions one needs three independent co-ordinates: rotation angles of half-clutches 1 and 2 and the centrifuge masses 4 distance from the rotation axle (Fig 2).

When the clutch does not rotate and ring 3 is not deformed, the distance of centrifugal mass 4 up to rotation centre 0 is equal to elastic ring radius $r$, and angle $\psi=90^{\circ}$ (Fig 2). When the system starts to rotate, the clutch under the action of load, opposing with a torsional moment, becomes deformed. The half-clutches of the clutch swing relatively to each other. This swing is characterized by a change of angle (by increase) (Fig 2). The deformation of ECRC $\Delta \psi=\left(\Delta \psi_{1}+\Delta \psi_{2}\right)$ shows itself as the deformation of the elastic ring and as a change of centrifugal masses distance from the rotation axis, approaching centre 0 (Fig 2). With increasing clutch rotation frequency, centrifugal forces of rotating masses 4 increase and partially bring masses 4 to initial position thus decreasing ERRC deformation.

Due to the intermediate deformation of the ring, rotation load is transformed into centrifugal forces caused by an elastic ring and rotating masses. At certain revolutions the system comes to a dynamic equilibrium which is characterized by the angle of deformation and the distance of centrifugal masses $\rho$ from the clutch rotation centre 0 . Let us examine some ECRC constructions.

Reverse ECRC with freely located rings, the axle of which is parallel to the axle of the shafts being connected and a geometrical centre of the ring is located on the rotation axle (Fig 4) [9-17]. ECRC, which main element being connected is a free elastic ring, is used to pass a torsional moment at not slight misalignment of connecting shafts and to suppress, at the same time, the rotational vibrations, dynamic interference and impacts.

Some modifications of the clutches (Fig 4 a, b, c, e) are introduced into hydraulic gears of metal-cutting machine tools, devices for measuring thread electric resistance and electrostatic charge, screw-drivers bolts and screw-nuts, gears of agricultural machines, etc. The clutch shown in Fig 2 e is covered by the patents in the USA, England and Germany [18-20].

Reverse ECRC with freely located rings the axle of which is perpendicular to the axle of the clutch consists of hub 1, the driven half-clutch of hub 2 passing into the drum (Fig 5 a) [21]. The half-clutches of the clutch are connected by elastic rings 3 evenly located in lengthwise splits of the half-clutches. To prevent falling out of the rings, naves 5 are used. The ring can be fixed by 
limiters 4. Splits are cut with the help of cylindrical milling cutters which thickness corresponds to the thickness of a glide matching elastic element. The size of axial misalignment of shafts can be regulated by the length of the driving half-clutch supporting split, which, in the cases of radial and angular misalignment, forms free cavity for the ring deformation and decreases additional loads of bearings.

The rings can be of various types (Fig $5 \mathrm{~b}, \mathrm{c}, \mathrm{d}, \mathrm{e}$ ): turned ring (b), winded ring (d) and Archimedean ring spiral in shape (c).

\section{Experimental research and practical application}

Test samples of the clutches were made and tested statistically and dynamically. The experimental sample of elastic centrifugal clutches (ECC) with a free ring is shown in Fig 6-8. Some tests of working-capacity and efficiency of the clutches were performed in the enterprises where the clutches were introduced into practice.

For the tests original stands created by the author were used. The construction of the stand was protected by the right of authorship.

The data of experimental research confirmed theoretical data. Discrepancy does not exceed $10 \%$.
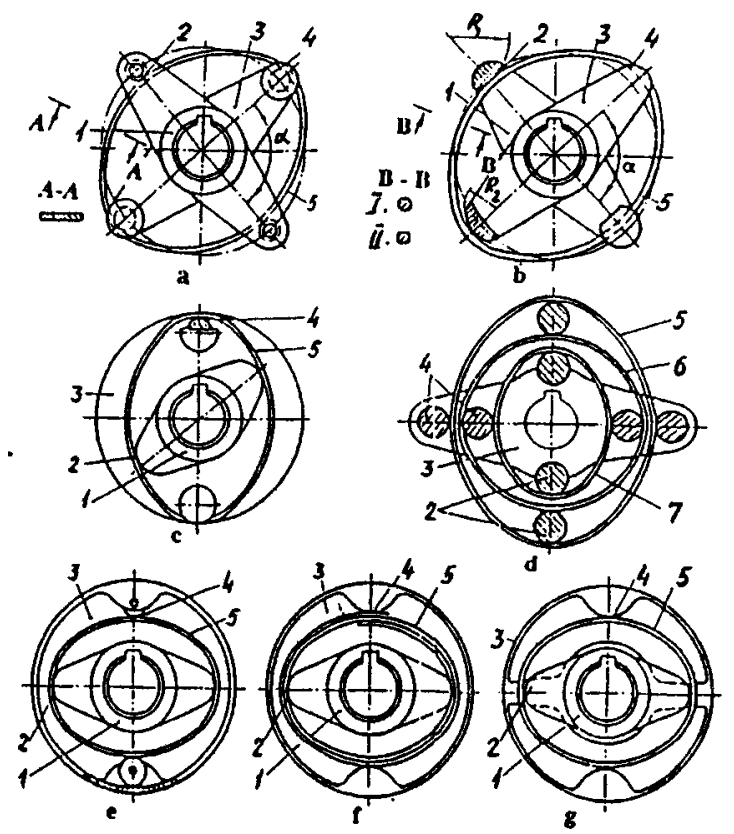

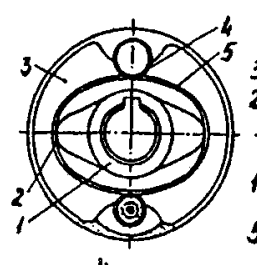

l.
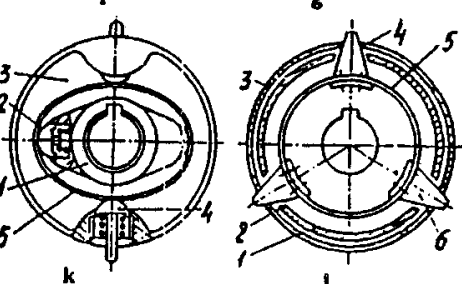

Fig 4. Scheme modifications of reverse ECRC with freely distributed rings
Rotational vibrations of the clutch were investigated with a D-21 stand. The excitation of the clutch by accidental interferences, properties of vibration isolation, etc. were studied.

Elastic centrifugal clutches with freely located rings, the axles of which are perpendicular to the rotation axle were designed, tested and introduced, according to a special program, into a gear of a hydraulic system instead of toothed clutches in the fodder production industry (Fig 6).

By experimental tests technical documents of the clutch type (Fig 7) were prepared and test samples made.

For mighty gears of oil pumps technical documents of the clutches were prepared and the clutches with elastic rings of Archimedean type spirals were made and tested (Fig 8). Such clutches improve vibration isolation reliability and exploitation properties of the gear as compared with earlier used toothed clutches [2].

Elastic centrifugal clutches with wound cylindrical springs located in one or two layers are widely used in oceanic or special ships. They are used both in drives of auxiliaries and in the main drives.
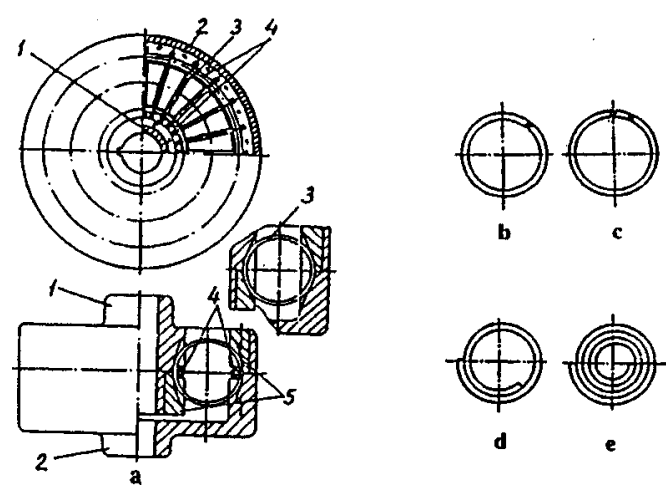

Fig 5. ECRC with freely located rings, which axle is perpendicular to the axle of the shafts being connected

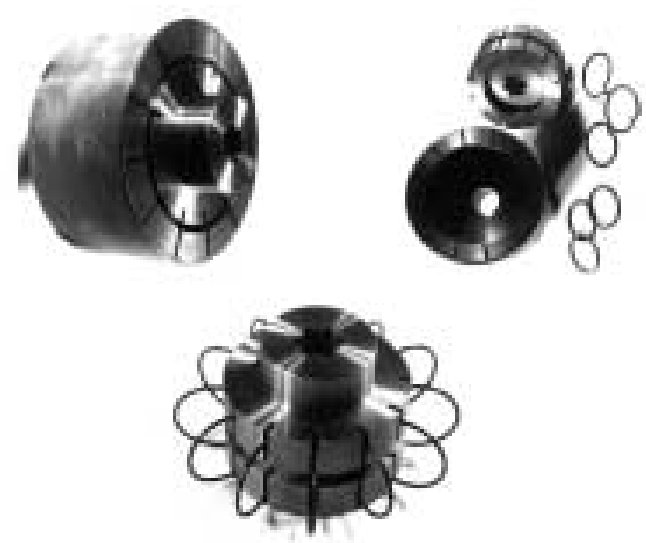

Fig 6. Clutch of foder crusher hydraulic gear: a - general view; $\mathrm{b}$ - composite parts of the clutch; $\mathrm{c}$ - driving halfclutch with rings located in slits 


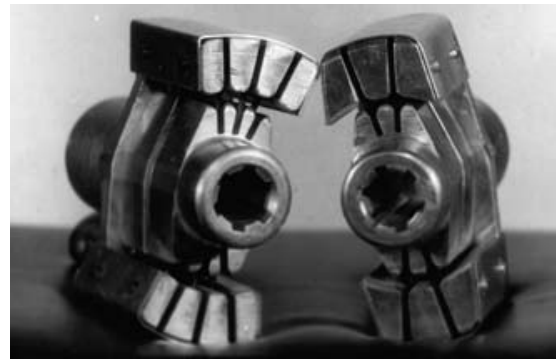

Fig 7. Fooder crusher hydraulic gear clutch with three (a) and two (b) pairs of rings

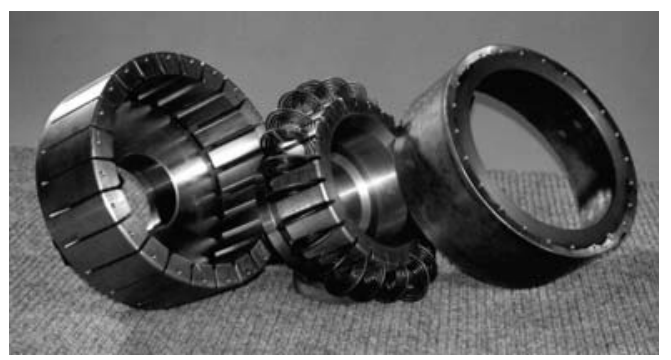

Fig 8. Composite elements of the clutch of oil pump gears

Radial rigidities of the clutch were calculated and hodographs of forces for various values of misalignment of the shaft axes $(e)$ and different numbers of rings $(N Z)$ (Fig 9) were presented.

As it is seen from Fig 9 when the connecting rings are of the same radial rigidities, the radial forces are larger for larger misalignment of shaft axes. Besides, the forces of radial rigidities are increased and the shape of stiffness hodograph of radial forces moves to the shape of circle increasing the number $(\mathrm{NZ})$ of the rings connecting the driving and the driven half-couplings [22-26].

Designs of the presented rotary motion transmission and stabilization devices are not complicated, but methodics of their calculation is rather difficult, because it is necessary to take into account all dynamical processes, misalignment of shaft axles, influence of highspeed drives centrifugal forces field, etc. That is why their practical application is effective when the method of parametric optimization of elastic elements of the devices, which estimates real working conditions of the object, is used. Such problems are discussed in scientific works of the author.

One of such clutches is used in special "Shkval" ship (Fig 10) for the connection of the main drive screw with $360 \mathrm{HP}$ diesel engine. The engine is fitted on elastic supports. The introduced clutch (instead of a tyre type clutch) considerably improved exploitation characteristics of the main drive: reduced the rotational vibrations of the system, reduced noises about $11 \mathrm{~dB}$, increased the exploitation resource of the drive by 1,7 times.

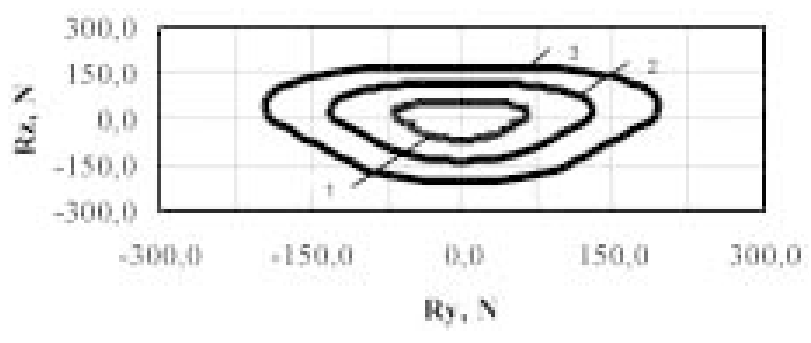

a)

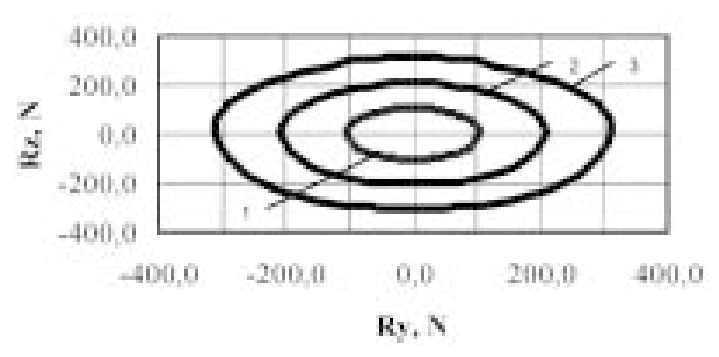

b)

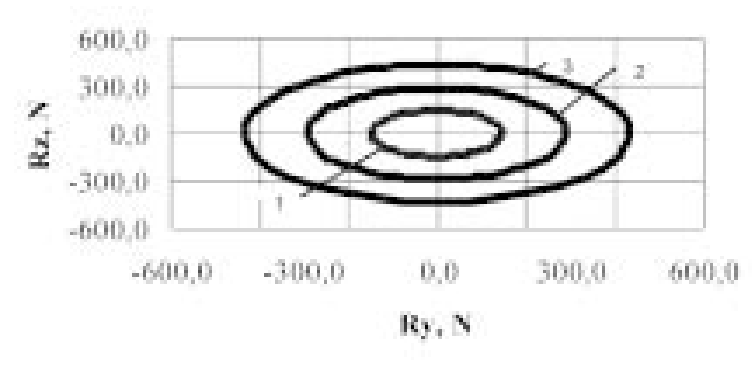

c)

Fig 9. Stiffness hodographs of radial forces: the clutch with a variable number of rings $(N Z)$ and misalignment of shafts axes $(e): a-N Z=3 ; b-N Z=5 ; c-N Z=7$; $1-e=0,5 \mathrm{~mm} ; 2-e=1,0 \mathrm{~mm} ; 3-e=1,5 \mathrm{~mm}$

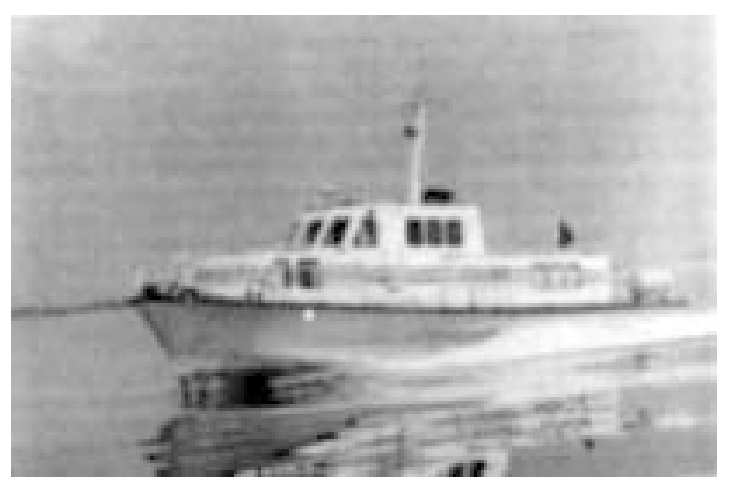

Fig 10. Special "Shkval" type ship 


\section{Conclusions}

1. To connect rotors, to transmit and stabilize rotation motion a new class of RMTSD was developed. They include the rings which deform axially, radially and at angle, the radial segments, etc. with joined concentrated inertial, dissipative and elastic elements. RMTSD design schemes are copyrighted and covered by the patents of the USA, England and Germany.

2. The classification of RMTSD is performed according to the following characteristic features: dynamic characteristics, kind of elastically creeping elements, peculiarities of design elements, fastening, materials. Etc.

3. The results obtained during laboratory studies of experimental samples of RMTSD and also exploitation tests of the clutches produced by industrial means enabled to introduce the RMTSD into practice in the following fields:

- devices of textile industry;

- ships of oceans, seas and in small boats;

- oil industry equipment;

- agricultural machines;

- auxiliary equipment of metal-cutting machines, etc.

\section{References}

1. Spruogis B. The divices of transmission and stabilization of rotary motion. Monograph (Устройства для передачи и стабилизации вращательного движения). Vilnius: Technika, 1997. 476 p. (in Russian).

2. Ragulskis K., Spruogis B., Ragulskis M. Transformation of rotational motion by inertia couplings. Monograph. Vilnius: Technika, 1999. $236 \mathrm{p}$.

3. Spruogis B. Rotary systems motion stabilization devices. In: Science and arts of Lithuania. Vibroengineering. Monograph. Vilnius, 1998, p. 452-465.

4. Spruogis B. Stabilizers of rotary motion in hydraulic drives. Transport Engineering (Transportas), No 2(11), Vilnius: Technika, 1995, p. 51-59 (in Lithuanian).

5. Spruogis B., Turla V. Original dynamic devices for translation and stabilization rotation movement. Engineering Physics (Инженерная физика), 2002, No 1, p. 11-14 (in Russian).

6. Spruogis B., Zubavičius L. Investigation of Movement of Couplings with Throttling Devices. Mechanics (Mechanika), No 6(32). Kaunas: Technologija, 2001, p. 40-46.

7. Spruogis B., Turla V. Drive and Stabilized Motion Devices in Rotary Systems. In: Agricultural Engineering (Žemès ūkio inžinerija. Mokslo darbai), No 33(3). Raudondvaris, 2001, p. 85-95 (in Lithuanian).

8. Spruogis B. Jurevičius J. Increase of Reliability of a Drive of the Conoidal Stone-Breaker at Reception of Broken Stone in the Construction of Highway. Transport Engineering (Transportas), Vol XIV, No 5. Vilnius: Technika, 1999, p.
245-252 (in Russian).

9. Kavolèlis A. P., Spruogis B. Centrifugal Coupling. USSR Patent No 361330. Inv. Bulletin 1, 1973.

10. Kavolèlis A. P., Spruogis B., Gulbinas A. Centrifugal Coupling. USSR Patent No 775462. Inv. Bulletin 10, 1980.

11. Kavolèlis A.P., Spruogis B. Centrifugal Coupling. USSR Patent No 868177. Inv. Bulletin 36, 1981.

12. Kavolèlis A. P., Spruogis B. Centrifugal Coupling. USSR Patent No 720234. Inv. Bulletin 9, 1980.

13. Kavolèlis A. P., Spruogis B., Naujokas R. Centrifugal Coupling. USSR Patent No 712560 Inv. Bulletin 4, 1980.

14. Kavolèlis A. P., Spruogis B., Solominas J. Centrifugal Coupling. USSR Patent No 641208. Inv. Bulletin 1, 1979.

15. Kavolèlis A. P., Spruogis B., Zubavičius L. Centrifugal Coupling. USSR Patent No 836416 Inv. Bulletin 21, 1981.

16. Kavolèlis A. P., Spruogis B. Centrifugal Coupling. USSR Patent No 853231 Inv. Bulletin 29, 1981.

17. Kavolèlis A. P., Spruogis B. Centrifugal Coupling. USSR Patent No 853232. Inv. Bulletin 29, 1981.

18. Kavolèlis A. P., Spruogis B., Jakštas A. and others. Centrifugal Elastic Coupling. United States Patent No 4118953. Washington, 1978.

19. Kavolèlis A. P., Spruogis B., Jakštas A. and others. Elastische Fliechkraffkuplung. Bundesrepublik. Deutschland. Deutsches Patentamt Offenlegungsschift DE 2700914A1. München, 1977.

20. Kavolèlis A. P., Spruogis B., Jakštas A. and others. Improvements in or Relating to Centrifugal Couplings. The United Kingdem of Great Britain. Patent No 1554801. London, 1977.

21. Spruogis B., and others. Centrifugal Coupling. USSR Patent No 1472716. Inv. Bulletin 14, 1989.

22. Bogdevičius M., Spruogis B. Theoretical investigations into rotary systems with elastic link caused by the deflection of the shafts. Transport Engineering (Transportas), No 2 (13), Vilnius: Technika, 1996, p. 70-81 (in Russian).

23. Bogdevičius B., Lingaitis L., Spruogis B. Dynamics and mathematical models of rotor system with flexible link in the presence of shafts misalignment. Mechanical Engineering (Inženyrska mechanika), 2003, No 4, Czech: Brno, p. 263-275.

24. Spruogis B., Turla V., Jurkonis E. Transmission devices for transferring and stabilization of rotational motion. In: International Conference "Power Transmission'03". Section I "Theory, Calculation and Design of Transmission Elements”. Bulgaria, Varna, 2003, p. 243-246.

25. Bogdevičius M., Spruogis B., Jurevičius J. Investigation of Rotor Systems with Elastic Links. In: Proceeding of The Fourth International Conference „Heavy machinery HM 2002“. Kraljevo, 2002, p. A.37-A.40 (in Russian).

26. Spruogis B., Turla V. Centrifugal Inertia Couplings with Free Rings which Axis are Perpendicular to the Rotation Axis of the Coupled Shafts. Journal of Vibroengineering, No 1 (10), Vilnius: Vibrotechnika, 2003, p. 15-18. 